\title{
Identifying facilitators and barriers to develop implementation strategy for an ED to Ward handover tool using behaviour change theory (EDWHAT)
}

\author{
Kate Curtis ${ }^{1,2,3^{*}}$, Tiana-Lee Elphick ${ }^{1} \mathbb{B}$, Madeline Eyles ${ }^{1}$ and Kate Ruperto ${ }^{1}$
}

\begin{abstract}
Background: Effective clinical handover is fundamental to clinical practice and recognised as a global quality and safety priority. Problems with clinical handover from the emergency department (ED) to inpatient ward across four hospitals in the Illawarra Shoalhaven Local Health District (ISLHD) were identified in a number of reportable clinical incidents. To address this, an ED to inpatient ward electronic clinical handover tool was developed and implemented. However, site uptake of the tool varied from 45 to $90 \%$.

Aim: To determine the facilitators and barriers of the ED to Ward Handover Tool (EDWHAT) implementation and design strategy to improve local compliance and inform wider implementation.

Methods: An exploratory convergent mixed-method approach was used. Data were collected via a 13-item electronic survey informed by the Theoretical Domains Framework (TDF) distributed to eligible nurses across the health district. Descriptive statistics for quantitative data and thematic analysis for qualitative data were conducted. The data were then integrated and mapped to the TDF and the Behaviour Change Wheel to identify specific behaviour change techniques to support implementation.

Results: There were 300 respondents. The majority of nurses knew where to locate the tool (91.26\%), but 45.79\% felt that it was not adequate to ensure safe handover. The most frequently reported factors that hindered nurses from using the tool were inability to access a phone near a computer (44.32\%) (environmental domain), being told to transfer the patient before being able to complete the form (39.93\%) (reinforcement) and the other nurse receiving (or giving) the handover not using the form (38.83\%) (social influence). An implementation checklist to identify barriers and solutions to future uptake was developed.

Conclusion: To improve uptake, the functionality, content, and flow of the handover tool must be revised, alongside environmental restructuring. Nurses would benefit from an awareness of each speciality's needs to develop a shared mental model and monitoring, and enforcement of tool use should become part of a routine audit.
\end{abstract}

Keywords: Behaviour change, Implementation, Clinical handover, Emergency, Nursing, Patient safety

\footnotetext{
* Correspondence: Kate.Curtis1@health.nsw.gov.au

${ }^{1}$ Illawarra Shoalhaven Local Health District, Wollongong Hospital, Loftus Street, Wollongong NSW 2500, Australia

${ }^{2}$ Susan Wakil School of Nursing and Midwifery, Sydney Nursing School, Faculty of Health and Medicine, The University of Sydney, 88 Mallett Street, Camperdown, NSW 2006, Australia

Full list of author information is available at the end of the article
}

(c) The Author(s). 2020 Open Access This article is licensed under a Creative Commons Attribution 4.0 International License, which permits use, sharing, adaptation, distribution and reproduction in any medium or format, as long as you give appropriate credit to the original author(s) and the source, provide a link to the Creative Commons licence, and indicate if changes were made. The images or other third party material in this article are included in the article's Creative Commons licence, unless indicated otherwise in a credit line to the material. If material is not included in the article's Creative Commons licence and your intended use is not permitted by statutory regulation or exceeds the permitted use, you will need to obtain permission directly from the copyright holder. To view a copy of this licence, visit http://creativecommons.org/licenses/by/4.0/ The Creative Commons Public Domain Dedication waiver (http://creativecommons.org/publicdomain/zero/1.0/) applies to the data made available in this article, unless otherwise stated in a credit line to the data. 


\section{Contributions to the literature}

- This original research work presents the facilitators and barriers of the ED to Ward Handover Tool (EDWHAT)

implementation and design strategy to improve local compliance and inform wider implementation.

- Behaviour change theory is applied to the emergency and acute care environment to find real-world practical solutions to improving clinical handover-a quality improvement priority.

\section{Background}

Effective clinical handover is fundamental to clinical practice and is recognised as a local [1], national [2], and global [3] quality and safety priority. Ineffective clinical handover increases the risk of patient-related errors as a result of poor communication and the lack of appropriate and timely transfer of critical information at the time of reporting. This creates inefficiencies in clinical practice leading to suboptimal patient care [2]. This is especially problematic at transitions of care, such as transfer from the ED to the ward, when communication errors are more likely and there is an increased risk of information being miscommunicated or lost. Ineffective communication at clinical handover is also associated with clinicians spending extensive time attempting to retrieve relevant and correct information [4]. This can result in inappropriate care, and the possibility of misuse or poor use of resources [5]. There remain multiple barriers to effective handover including a lack of systematic structure, including incomplete handovers, excessive reliance on memory without reference to written documentation and poor quality of written medical records [6]. These barriers are particularly prevalent in the complex and hectic emergency department (ED) context where more than 2.5 million ED to ward clinical handovers occur in Australian hospitals each year [7], and the work pattern is unpredictable making delivery of safe patient handover challenging [8]. Responses to this have included recommendations to standardise the structure of handover communication [9] and include the patient and their carers in the handover [2]. An effective standardised and structured clinical handover process enhances the reliability of information transfer by decreasing the reliance on memory and maintaining a focus on important aspects of care [10]. All relevant participants know the minimum information that needs to be communicated when handovers take place, the purpose of the handover, the structured format to aid communication, and how responsibility and accountability are transferred. Further, critical information is more likely to be accurately transferred and acted on. Standardised and structured clinical handover results in greater levels of patient satisfaction [11], better understanding of diagnosis and treatment [12], and reduced errors $[6,13]$.

The clinical handover from the ED to inpatient ward across four hospitals in the Illawarra Shoalhaven Local Health District (ISLHD) was identified by incident management system reports to be inadequate. The clinical handover practice was not meeting key criteria of the local Ministry of Health policy Clinical Handover [1], particularly the requirement of a standardised process as different groups of clinicians were requiring different information resulting in varying quality of the information provided. This coincided with a number of reportable clinical incidents, and subsequent recommendations to improve the standard of ED to ward clinical handover practices.

To address this policy-practice gap, an ED to inpatient ward clinical handover tool (EDWHAT), was developed to comply with the Ministry of Health Clinical Handover policy and for the use in the digital platform used by the ISLHD (Fig. 1). EDWHAT was developed by an ED and ward nurse educator and nurse manager. The tool was reviewed by a small group of senior nurses and then implemented at all four hospital sites by local nurse educators using face to face education. A formal strategy was not developed. A local audit following the implementation of EDWHAT demonstrated that uptake of the tool was mixed between sites and wards. Compliance at the different sites ranged from 45 to $90 \%$ (mean $70.1 \%)$. The proposed research will determine the facilitators and barriers to the use of this newly implemented clinical handover tool by nursing staff at the study sites. Behaviour change theory will then be used to develop interventions to improve the target behaviour, that is, the use of the clinical handover tool.

\section{Methods}

This exploratory convergent mixed-method study [14] was designed using French et al.'s four-step implementation process model [15]; (a) clarifying who needs to do what differently (nursing staff), (b) identifying barriers and enablers (electronic survey of nursing staff, informed by the Theoretical Domains Framework), (c) selecting fit-forpurpose intervention strategies and components (mapping survey results using the behaviour change wheel) and (d) evaluating implementation interventions (comparing the original implementation with the findings of this study). The study process is outlined in Fig. 2. The study was conducted in a Local Health District (LHD) which has four geographically and casemix diverse EDs (rural, regional, metropolitan). The STROBE Statement was used as a checklist for reporting guidelines for cross-sectional studies and is provided as Additional file 1. Research conducted as part of this study adhered to the National Statement on the Conduct of Human Research by the 


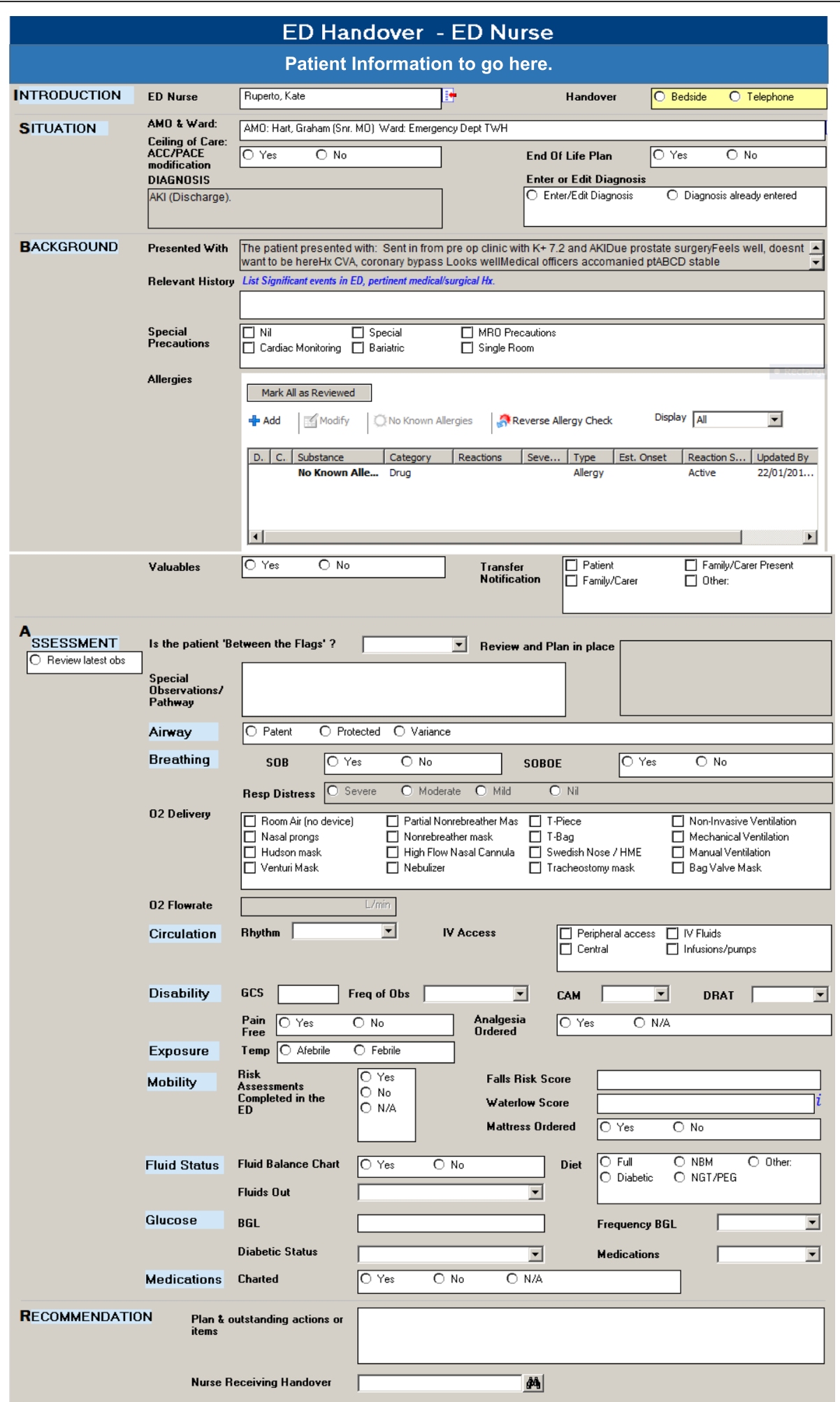

Fig. 1 The ED to Ward electronic handover tool 


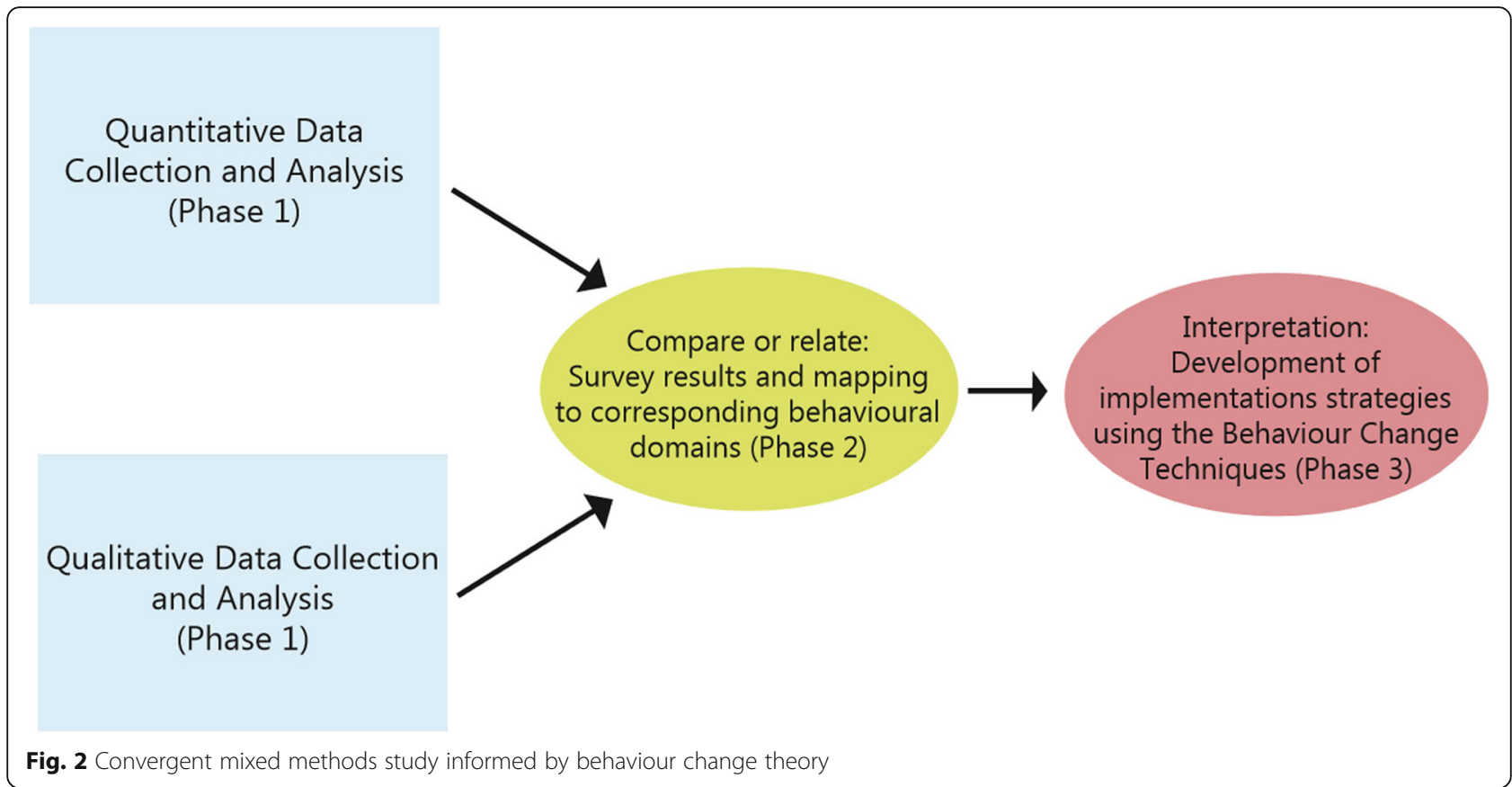

Australian National Health and Medical Research Council and was approved by the University of Wollongong (UOW) and ISLHD Health and Medical Human Research Ethics Committee (LNR/18/WGONG/148).

\section{Phase 1: staff survey Instrument}

The electronic survey was administered via REDCap (Research Data Capture), a secure Web-based application for data management and survey tool. The survey commenced with participant characteristics such as age, place of work (ward or ED) and level of experience. This was followed by 11 questions focussed on known barriers and enablers to uptake of new tools in the clinical environment generally [2] and specific to the study site $[16,17]$ that were then mapped to the domains of the Theoretical Domains Framework (TDF). The TDF is a synthesis of behavioural change theories presented as a framework to explore the science of intervention implementation in health care [18]. The TDF was selected to inform the survey to enable targeted questions related to practice within the clinical environment, classification of enablers and barriers using a broad range behavioural influences, as the investigators had previously successfully used the TDF to identify and design interventions in the ED/acute care context [16, 17, 19]. Each of the key influences on behaviour are listed in Table 1. The information gathered from the survey assisted in identifying the main factors influencing behaviour change in the clinical setting. Most questions had a 5-point Likert response, with the opportunity for free text/comment. The survey can be found in Additional file 2 .

\section{Participant recruitment and procedure}

Eligible participants were registered or enrolled nurses, who as part of their role would be involved in the giving or receiving of handover on patients being transferred from an ISLHD ED to a participating inpatient unit within the ISLHD. Maternity and mental health units were excluded as at the time of the study, they did not use the handover tool. There were approximately 3000 nurses across ISLHD eligible to complete the survey.

The study was conducted over a 5-month period from November 2018 to March 2019. The data collection period was extended due to delays in distribution and the Christmas holiday period. Participants were emailed the survey link and a participant information sheet to their NSW Health staff email address via their managers. Time to complete the survey was allocated during education and other downtimes. Reminders were sent fortnightly.

\section{Data analysis}

Quantitative survey data were analysed using descriptive statistics. Preliminary analyses were run to check for missing data. Responses were presented as proportional data. Sub-analyses were conducted to identify specific barriers or facilitators by site, and/or by ward or ED location. Qualitative data from the free-text responses were analysed using inductive thematic analysis using Braun and Clark's 6-step process [21]. The responses were read through several times to get a sense of the data and allocated into thematic areas [22]. The meaning units were developed by condensing the text. Meaning units were classified into themes and sub-themes. This process was completed by two authors (KR and KC). 
Table 1 The 14 behavioural influences of the Theoretical Domains Framework [20]

\section{Domains included in the survey are italicised}

1. Knowledge (An awareness of the existence of something)

2. Skills (An ability or proficiency acquired through practice)

3. Social/professional role and identity (A coherent set of behaviours and displayed personal qualities of an individual in a social or work setting)

4. Beliefs about capabilities self-confidence (Acceptance of the truth, reality, or validity about an ability, talent, or facility that a person can put to constructive use)

5. Optimism (Confidence that things will happen for the best or desired goals will be attained)

6. Beliefs about consequences (Acceptance of the truth, reality, or validity about outcomes of a behaviour in a given situation)

7. Reinforcement (Increasing the probability of a response by arranging a dependent relationship, or contingency, between the response and a given stimulus)

8. Intentions (A conscious decision to perform a behaviour or a resolve to act in a certain way)

9. Goals (Mental representations of outcomes or end states that an individual wants to achieve)

10. Memory, attention and decision processes (The ability to retain information, focus selectively on aspects of the environment and choose between two or more alternatives)

11. Environmental context and resources (Any circumstance of a person's situation or environment that discourages or encourages the development of skills and abilities, independence, social competence, and adaptive behaviour)

12. Social influences (Interpersonal processes that can cause individuals to change their thoughts, feelings, or behaviours)

13. Emotion (A complex reaction pattern, involving experiential, behavioural, and physiological elements, by which the individual attempts to deal with a personally significant matter or event)

14. Behavioural regulation (Anything aimed at managing or changing actions)

Phase 2: identification of facilitators and barriers and data integration

Quantitative items were considered facilitators if they were positively worded with greater or equal to $70 \%$ agreement with a statement. Items were considered barriers if they were positively worded with less than $70 \%$ agreement with a statement [23, 24]. Qualitative themes were considered facilitators or barriers based on the literature, and/or positively or negatively worded statement. The quantitative and qualitative results were then mapped to each of the TDF domains by two authors ( $\mathrm{KR}$ and $\mathrm{KC}$ ). Each item was reviewed to identify potential facilitators and barriers to implementation in each domain. If it was unclear if an item (survey response or theme) was a facilitator or barrier for the implementation, then the consensus was sought from a senior member of the study team with experience in the use of the TDF (BM). For any items that crossed multiple domains, for ease of presentation, we selected the domain we felt most represented the item. However, if the secondary domain was not identified in any other items, we allocated that domain to prevent the omission of any relevant domains. Sub-analyses (cross-tabulation) of enablers and barriers were conducted to determine if there were any findings specific to the type of nurse (ED or ward) or hospital site.

\section{Phase 3: development of implementation strategies}

The facilitators and barriers from the survey were then mapped to intervention functions and behaviour change techniques guided by the Behaviour Change Wheel [25]. Firstly, the authors mapped the TDF domains identified in phase 2 to intervention functions. Intervention functions are "broad categories by means of which an intervention can change behaviour". Each intervention function was assessed to see if they were Affordable, Practical, Effective, Acceptable, had Side-effects and were safe and Equitable (APEASE criteria) [25], and prioritised [26]. Stakeholders (front line nurses, nurse managers, nurse educators) were involved in the assessment of intervention functions during a series of meetings where the initial concept was tabled at various departmental meetings to garner support for the intervention. Secondly, the authors chose behaviour change techniques (BCTs) from the Behaviour Change Technique Taxonomy (BCTTv1) based on the intervention functions. A BCT is a component of an intervention that will alter behaviour [25]. The taxonomy includes 93 techniques for behaviour change linked to the Behaviour Change Wheel. Each intervention function is associated with a list of BCTs that are relevant to that intervention function, which can be assessed for relevancy to the local context. For example, if the barriers and facilitators differed between the ward and emergency nurses, the intervention function and supporting BCTs were refined to that context. Each BCT was also assessed using the APEASE criteria for inclusion alongside the intervention functions. The resulting BCTs were collated and used as suggested techniques to support the selected modes of delivery specific to the context of the sites (wards vs EDs, or particular sites). The TDF domains were grouped using COM-B then developed to a logic map.

\section{Results}

\section{Participant characteristics}

Of the 300 nurses (approximate10\% response rate from the whole of ISLHD) involved in the study, $80.41 \%$ were between 20 and 50 years of age, with just over one third (33.89\%) between six and 15 years of experience. The responses were proportionally representative of the size of each site. Just over half of respondents were ward nurses (56.7\%) (Table 2). 
Table 2 Characteristics of nursing respondents

\begin{tabular}{ll}
\hline Participant characteristic & Number (\%) $(\boldsymbol{N}=301)$ \\
\hline Hospital location* & $142(47.3)$ \\
Wollongong Hospital (WH) & $96(32.0)$ \\
Shoalhaven District Memorial Hospital (SDMH) & $51(17.0)$ \\
Shellharbour Hospital (SHH) & $11(3.7)$ \\
Milton Ulladulla Hospital (MUH) & \\
Nurse type* & $158(56.7)$ \\
Ward & $123(41.0)$ \\
ED & $19(6.3)$ \\
Both & \\
Age group & $1(0.3)$ \\
$<20$ years & $77(25.6)$ \\
$20-30$ years & $86(28.6)$ \\
$31-40$ years & $70(23.3)$ \\
$41-50$ years & $57(18.9)$ \\
$51-60$ years & $10(3.3)$ \\
$61-70$ years & $0(0.0)$ \\
$>70$ years &
\end{tabular}

\section{Reasons for not using the EDWHAT}

The most common reasons for not using EDWHAT were uniform across nurse type and hospital site. However, within each reason, there was some variance. For example, the most frequently cited reason for not using the tool was the inability to locate a computer near a phone $(44 \%, n=121$, but more so in the ward $47 \%, n=$ 65), being told to transfer the patient before being able to complete the form (particularly in ED 52.10\%, $n=$ 62 ), the other nurse receiving (or giving) the handover not using the form $(38.83 \%, n=106)$ and not having time $(34 \%, n=94)$ (Fig. 3). More than half of nurses from the two sites with the poorest uptake felt the tool did not support timely $(60 \%, n=75 ; 55 \%, n=50)$ or easy handover ( $54 \%, n=68$ and $54 \%, n=49)$. These two sites also had the largest proportions of nurses that reported being told to transfer the patient before being able to complete the form (33\%, $n=42$ and $55 \%, n=$ $50)$. The site who had the highest uptake also had the highest proportion of nurses reporting the tool was important $(76 \%, n=38)$.
Quantitative results presented within the corresponding behavioural domains

\section{Knowledge}

Knowing where to locate the tool proved a facilitator to using the tool, with $91.26 \%(n=261)$ of staff across all nursing types knowing where to locate the tool. All of the nurses in the ED knew where to locate the tool, whereas a small portion of ward nurses did not $(16 \%, n=24)$.

\section{Skills}

The majority of nurses $(78.25 \%, n=223)$ indicated they had a good understanding of how to use the tool. Some ward nurses however had little $(23.13 \%, n=34)$ or no $(8.16 \%, n=12)$ understanding of how to fill out the tool, which was considered a barrier.

\section{Environment}

Environmental influences were a substantial barrier. The most frequently reported factor that hindered nurses from using the tool was the inability to access a phone near a computer $(44.32 \%, n=121)$.

\section{Beliefs about capabilities}

The majority of respondents reported they felt confident using the tool $(77.39 \%, n=219)$. However, more ward nurses reported not feeling confident with using the tool (33.10\%, $n=48)$ compared to ED nurses $(9.92 \%, n=12)$. Overall, $68.27 \%(n=185)$ of nurses agreed that the tool was easy to use. Within the ED setting, ease of use was a facilitator $(78.99 \%, n=94)$, whereas this was a barrier for ward nurses $(60.74 \%, n=82)$ and nurses who work in both contexts $(52.94 \%, n=9)$.

\section{Beliefs about consequences}

Beliefs about consequences were one of the largest barriers to use of the tool, as just over half of the respondents considering the tool important (59.30\%), in particular those aged over 51 years. The age group with the largest proportion reporting that the tool was not important was the 31-40-year group (46.27\%). There was a little difference between ED and ward nurses regarding the importance $(59.86 \%$ and $57.85 \%$ ) or how long nurses had been working. The information communicated within the tool was also a substantial barrier as nearly half of respondents felt it was inadequate to ensure safe handover $(45.79 \%, n=125)$. The majority of nurses considered the tool did not promote timely handover $(54.91 \%, n=151)$ or make the handover process easier $(50.91 \%, n=140)$. These beliefs trends are the same between nurses who work on the wards, in the ED, and who do both. 


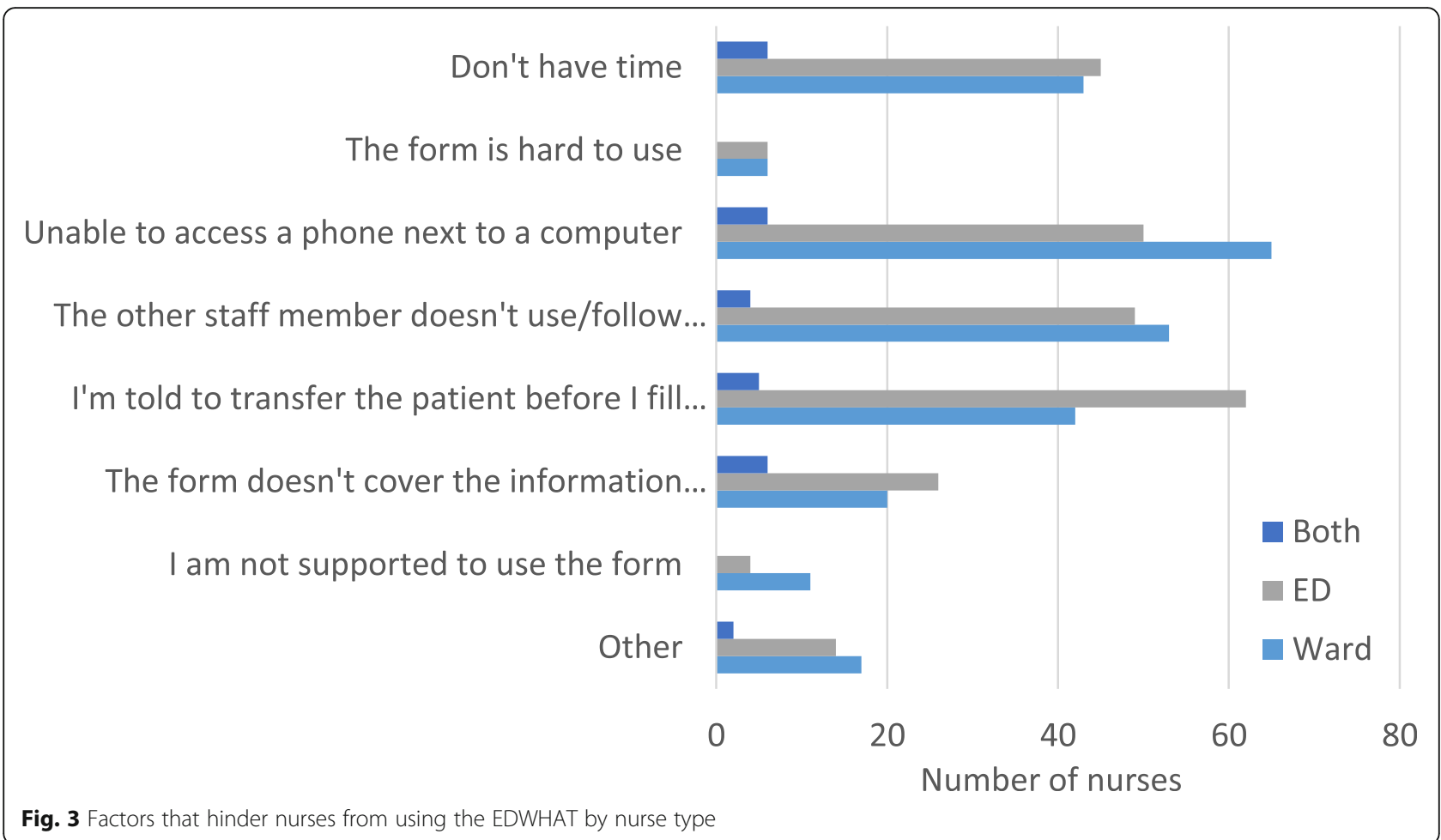

\section{Memory}

Overall, finding the tool easy to remember to use was a facilitator $(83.15 \%, n=232)$. However, this trend differed between nurse types, with this being a barrier for ward nurses $(51.05 \%, n=73)$ in contrast to ED nurses $(81.51 \%, n=97)$. The greatest factor for remembering to use the tool is having time to prepare for the handover (52\%), which is similar between nurse types. In contrast, the factor contributing the least to remembering to use the tool is being reminded by a manager (6\%) (Fig. 4).

\section{Qualitative results}

There were 399 free-text comments, resulting in 31 themes representing 22 barriers and nine facilitators (Table 3). These mapped to eight of the 14 TDF domains (Table 3 ). The most barriers were related to the environment in which the tool is required to be used, the content and usability of the form, and beliefs about the benefits (or lack thereof), particularly in relation to social influence. The most problematic behavioural influence was the environment with four sub-themes. Firstly, the functionality of the form within the electronic medical record system. This highlighted the technical difficulties of having the information in a "form" template within the electronic medical record, which means it cannot be minimised or open and shut, without losing information, to view other areas of the patient chart. Encapsulated by these respondents;
"Issue is that once in the tool you cannot revisit your notes which is a problem because you miss vital information" (ED nurse 16)

"Access to the form should be instant as ED/ward are very busy. Access to the handover form depends on computer availability, staff login time and form loading time, for a busy ward and at ED the wait is too long. Also some ED Nurses are happy to wait for form to be accessed, some ensure you hear their displeasure with sighs or statements of being too busy to wait." (Ward nurse 301)

"...The form is hard to access, through 3 menu's..." (Ward nurse 222)

Secondly, there were a number of responses related to the lack of phones and computers which impacted on staff ability to use the form to handover. For example,

"The only reason to not fill the form out is access to computers/ telephones" (ED nurse 204)

and;

"there have been times where all the computers have been used and there is no other option than to write on a piece of paper....buy more computers" (Ward nurse 78) 


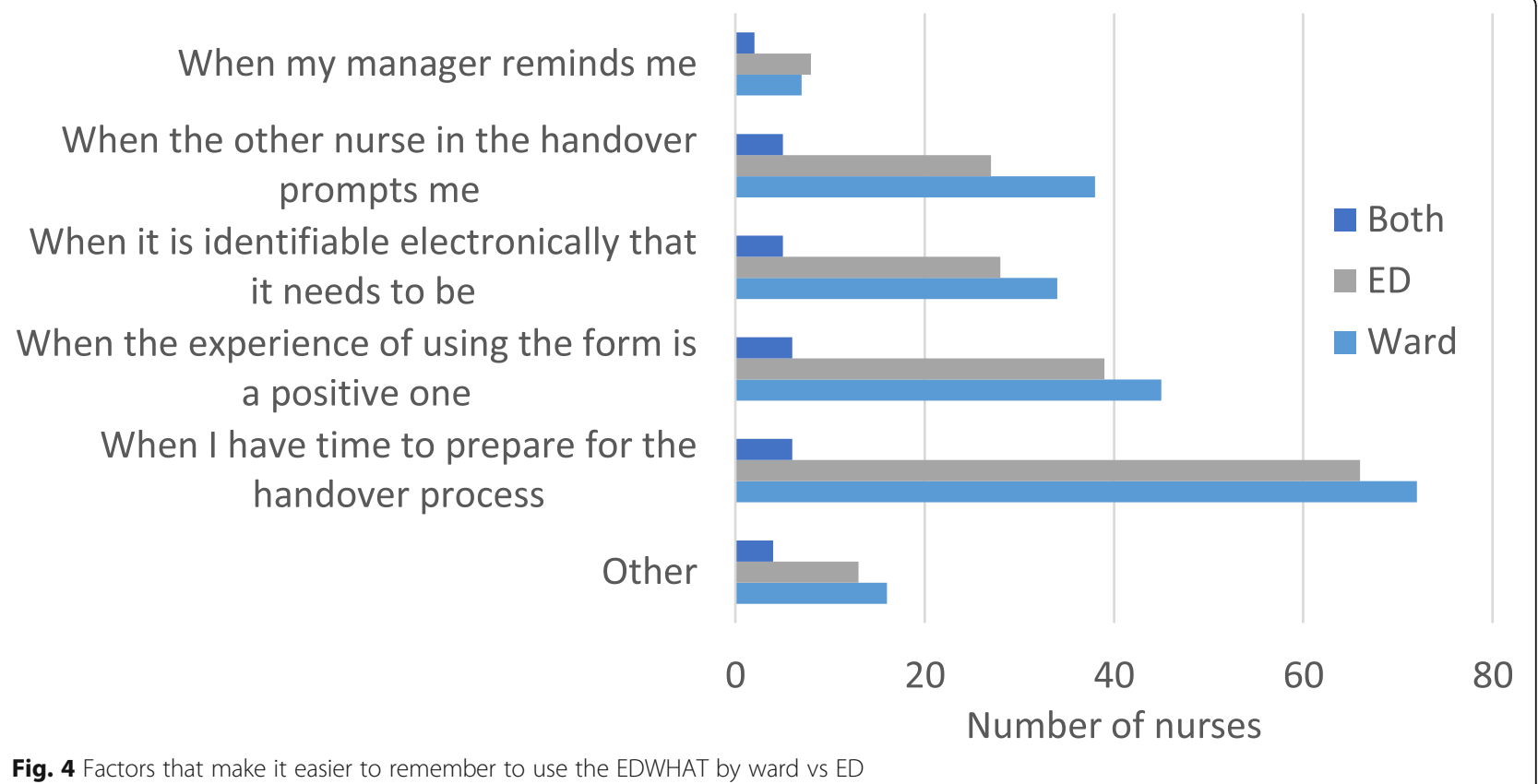

The third sub-theme identified concerns related to the structure of the form. The form has limited free-text sections, some auto-populated information, with the majority of information being tick boxes. A number of comments are related to the content of the form itself; however, this was often contradictory. For example, these respondents stated;

"I believe that the section where you tick that their obs are btf is important to ensure safe transfer as well as outstanding actions to ensure there is a record of what still needs to be done for the patient" (ED nurse 17)

"It is easy to use however it is useless as it does not provide enough information and the other staff member becomes complacent and just wants to tick boxes" (ED nurse 158)

And in contrast;

"sometimes important information is lost or importance negated by the volume of information asked for and given. often questions are mechanically asked with nil active listening involved..." (ED nurse 24)

"...Some nurses will ask all the questions in the tool and then they go to hang up, and you have to promt them and say ' do you wan tto know what i have done since they have been here now?"' (ED nurse 249)

The other frequently reported domains were beliefs about consequences and social roles and influences.
Within these domains, staff reported feeling that the tool was useful in helping important patient safety information gets handed over and improving the patient continuity of care and the patient experience;

"The tool is useful in ensuring a holistic handover is covered" (ED nurse 204)

However, responses also highlighted a number of beliefs held by the nurse sending the patient (ED) and the nurse receiving the patient (ward) about the handover. These beliefs seem to indicate conflict which may have been present prior to the introduction of the form. This may indicate differences in the working environments of emergency departments and wards who have different functions and responsibilities. Responses from ward staff suggest a lack of knowledge about the patient by ED staff;

"most of the time handover was wrong and wasting time filling the form" (Ward nurse 280)

and;

"...The ED nurses most of the time do not know enough information about the patients to be able to complete all the questions, as they are taking over from another nurse who cared for that patient previously or it is another nurse bringing the patient to the ward. A telephone handover from the nurse who is caring for the patient, so I can access my computer and they can access their computer to complete the form and then the ward nurse collects the patient or 
Table 3 The 32 themes identified that facilitate or hinder the use of the EDWHAT in the ISLHD

\begin{tabular}{|c|c|}
\hline Barriers & TDF domain \\
\hline Not supported by manager to complete handover & Reinforcement \\
\hline Not being face to face for handover means things get missed & Beliefs about consequences \\
\hline Doesn't make a difference to the handover I get & Beliefs about consequences \\
\hline ED staff don't use properly and don't like using it & Social influence \\
\hline Ward staff don't want to use it & Social influence \\
\hline Can't click out of the from to other part of the notes & Environment \\
\hline Duplication of processes & Environment \\
\hline Results in a focus on tick boxes and not the whole patient story & Environment \\
\hline Irrelevant content within the form & Environment \\
\hline Cumbersome & Environment \\
\hline Difficult to locate form in eMR & Environment \\
\hline Time restrictions-it takes too long & Environment \\
\hline Pt in transfer before hand over starts & Environment \\
\hline Unavailability or difficult location of computer & Environment \\
\hline Structure/flow is not intuitive & Environment \\
\hline More information is required than listed in the form & Environment \\
\hline Time pressure to move patients does not support handover & Environment \\
\hline Hard to remember to use & Memory \\
\hline Usefulness is user dependent & Skills \\
\hline Minimal use means unfamiliarity & Skills \\
\hline Has to be mutual agreement between ward and ED to complete & Social/professional role \\
\hline ED don't know their patient & Social influences \\
\hline \multicolumn{2}{|l|}{ Facilitators } \\
\hline Ensures completion of tasks & Beliefs about consequences \\
\hline Important for patient safety & Optimism \\
\hline Improves communication between ED and ward & Beliefs about consequences \\
\hline Improves continuity of care & Beliefs about consequences \\
\hline Provides evidence of handover & Beliefs about consequences \\
\hline Good systematic tool that ensures everything completed & Beliefs about consequences \\
\hline Prompts identification of issues & Beliefs about consequences \\
\hline Prompts sharing of the information needed for handover & Beliefs about consequences \\
\hline It's just part of what we do & Social/professional role \\
\hline
\end{tabular}

the ED nurse brings the pateint down would be MORE EFFECTIVE." (Ward nurse 222)

Conversely, the ED nurse respondents felt the ward staff are focused on tasks and not the patient condition;

"Often ward nurses want to skip the brief handover (2 minutes). I would usually provide that tells them why the patient presented, what we've done and the plan. They only want to hear the answers to the questions prompted by the form" (ED nurse 97)
Within these themes, time pressure was raised as a strong influence on the ability to adequately handover,

"Being told to hurry a handover does not respect the value of handover" (Ward nurse 10)

and;

"I don't have any objection to using the handover form. However I have found time constraints sometimes prevent me using it. Also at times we are directed to transfer the patient prior to having handover" (Ward nurse 81) 
Table 4 TDF domains identified to contain facilitators and barriers mapped to nine intervention functions and BCTS with APEASE

\begin{tabular}{|c|c|c|c|c|c|c|c|c|c|c|}
\hline & Education & Persuasion & Incentivisation & Coercion & Training & Restriction & $\begin{array}{l}\text { Environmental } \\
\text { restructuring }\end{array}$ & Modelling & Enablement & $\begin{array}{l}\text { BCTTV1 } \\
\text { selected }\end{array}$ \\
\hline Knowledge & $\checkmark$ & & & & & & & & & $2.2,5.1,5.3,7.1$ \\
\hline Skills & & & & & $\checkmark$ & & & & & $2.2,4.1,6.1$ \\
\hline $\begin{array}{l}\text { Beliefs about } \\
\text { capabilities }\end{array}$ & $\checkmark$ & $\checkmark$ & & & $\checkmark$ & & & $\checkmark$ & & $\begin{array}{l}2.2,4.1,5.1,5.2 \\
5.3,6.1,6.2,9.1 \\
13.1,15.1\end{array}$ \\
\hline $\begin{array}{l}\text { Social/ } \\
\text { Professional } \\
\text { Role and } \\
\text { Identity }\end{array}$ & $\checkmark$ & $\checkmark$ & & & & & & $\checkmark$ & & $\begin{array}{l}2.2,5.1,5.2,5.3 \\
6.1,6.2,9.1 \\
13.1,15.1\end{array}$ \\
\hline Optimism & $\checkmark$ & $\checkmark$ & & & & & & $\checkmark$ & $\checkmark$ & $\begin{array}{l}2.2,3.1,5.1,5.2 \\
5.3,6.1,6.2,9.1 \\
12.1,12.5,13.1 \\
15.1\end{array}$ \\
\hline $\begin{array}{l}\text { Beliefs about } \\
\text { Consequences }\end{array}$ & $\checkmark$ & $\checkmark$ & & & & & & $\checkmark$ & & $\begin{array}{l}2.2,5.1,5.2,5.3 \\
6.1,6.2,9.1 \\
13.1,15.1\end{array}$ \\
\hline $\begin{array}{l}\text { Memory, } \\
\text { Attention } \\
\text { Decision } \\
\text { Processes }\end{array}$ & & & & & $\checkmark$ & & $\checkmark$ & & $\checkmark$ & $\begin{array}{l}2.2,3.1,4.1,5.1 \\
6.1,7.1,12.1 \\
12.5,13.115 .1\end{array}$ \\
\hline $\begin{array}{l}\text { Environmental } \\
\text { Context } \\
\text { Resources }\end{array}$ & & & & & $\checkmark$ & $\checkmark$ & $\checkmark$ & & $\checkmark$ & $\begin{array}{l}2.2,3.1,4.1,6.1 \\
12.1,12.5,13.1 \\
15.1\end{array}$ \\
\hline $\begin{array}{l}\text { Social } \\
\text { Influences }\end{array}$ & & & & & & $\checkmark$ & $\checkmark$ & $\checkmark$ & $\checkmark$ & $\begin{array}{l}2.2,3.1,4.1,5.1 \\
6.1,7.1,12.1 \\
12.5,13.1,15.1\end{array}$ \\
\hline Reinforcement & & & $\checkmark$ & $\checkmark$ & $\checkmark$ & & $\checkmark$ & & & $\begin{array}{l}2.1,2.2,4.1,5.1 \\
6.1,10.4,10.5, \\
12.1,12.5\end{array}$ \\
\hline
\end{tabular}

\section{Intervention Functions and comments around APEASE criteria}

Education Education is affordable and practical within existing roles and orientation processes, however Excessive educational focus may lead to neglect in other domains. Educational meetings alone are unlikely to effectively change complex behaviours [26]. This was the main intervention in initial implementation that had suboptimal uptake

Persuasion Using persuasive communication to induce positive feelings to stimulate use of EDWHAT by senior staff, managers and peers all existing roles. But need to consider method of persuasion not to be construed as "pushy". Needs to be conducted equitably - not single out particular staff

Incentivisation Creating an expectation of award (incentive) is a crucial characteristic for the intervention, given the competing demands on nurse time, and the lack of existing recognition for use of the tool. Financial incentives are not affordable or practical, however awards, recognition and positive feedback may be effective. Can be seen as "core business so shouldn't need incentive". Care must be taken that the incentive chosen must be widely available - all staff have opportunity for acknowledgement

Coercion Creating expectation of punishment or cost through coercion while affordable and possible within existing roles, is not appropriate

Training Imparting skills is affordable and practical within existing roles and orientation processes but staff need opportunity and motivation to use them

Restriction Using rules to increase staff using the tool by reducing competing behaviours is possible within existing resources and structure. Particular care to be taken to ensure equitable reinforcement for all staff

Environmental Changing the physical context is crucial. It can be conducted with existing IT and senior nursing staff roles. Additional computers are not an restructuring onerous budget item and a priority

Modelling Providing an example for people to aspire to or imitate by modelling is affordable, practical, effective, acceptable and equitable within existing roles and orientation processes.

Enablement Increasing the means and reducing barriers to increase capability and opportunity for staff to use the tool through enablement Affordable, practical, effective, acceptable and equitable within existing roles and orientation processes.

Selected BCTs 2.1 monitoring of behaviour by others without evidence of feedback; 2.2 feedback on behaviour; 3.1 social support (unspecified); 4.1 instruction on how to perform a behaviour; 5.1 information about health consequences; 5.2 salience of consequences; 5.3 information about social and environmental consequences; 6.1 demonstration of the behaviour; 6.2 social comparison; 7.1 prompts/cues; 9.1 credible source; 10.4 social reward; 10.5 social incentive; 12.1 restructuring the physical environment; 12.5 adding objects to the environment; 13.1 identification of self as role model; 15.1 verbal persuasion about capability. 
"The information is only as good as what is handed over from ED. It can vary from thorough, to vague, to someone who doesn't know the patient. An experienced clinician knows the questions to ask, a novice clinician may take all information on face value" (Ward nurse 36)

\section{Phase 3: development of implementation strategies}

The TDF domains identified in phase 2 were mapped to the nine corresponding intervention functions (Table 4). Each intervention function [27] and selected BCT [28] was assessed using the APEASE criteria. Seven of the nine intervention functions and 18 BCTs were considered appropriate for use in future implementation, and the commentary from key stakeholders regarding each intervention function is listed in Table 4.

The agreed-upon BCTs were collated and were used to develop implementation strategies (modes of delivery) specific to the context of the sites (wards vs EDs, or particular sites). These were then developed into a logic map (Fig. 5). The logic map demonstrates the mapping process and selection of BCTs to inform the strategy to improve uptake of the EDWHAT. Filled boxes or partially filled boxes in the recommendation column represent interventions/BCTs that were completed or partially completed in the original implementation. An implementation checklist for future use was developed based on the behavioural influences most prominent in the survey responses and themes, the intervention functions selected through the APEASE process and gaps highlighted by the logic map (Fig. 6).

\section{Discussion}

This study demonstrated the numerous barriers and facilitators to uptake of a new intervention designed to improve ED to Ward Handover across a local health district, in particular, the influence of the working environment, social influence and beliefs about consequences.

Implementation of new interventions needs planning and strategy that address the complexity of healthcare systems and individual practitioners [29] and strong organisational support [30]. There are multiple models available on which to develop and plan an implementation strategy [31], the most well known in health is

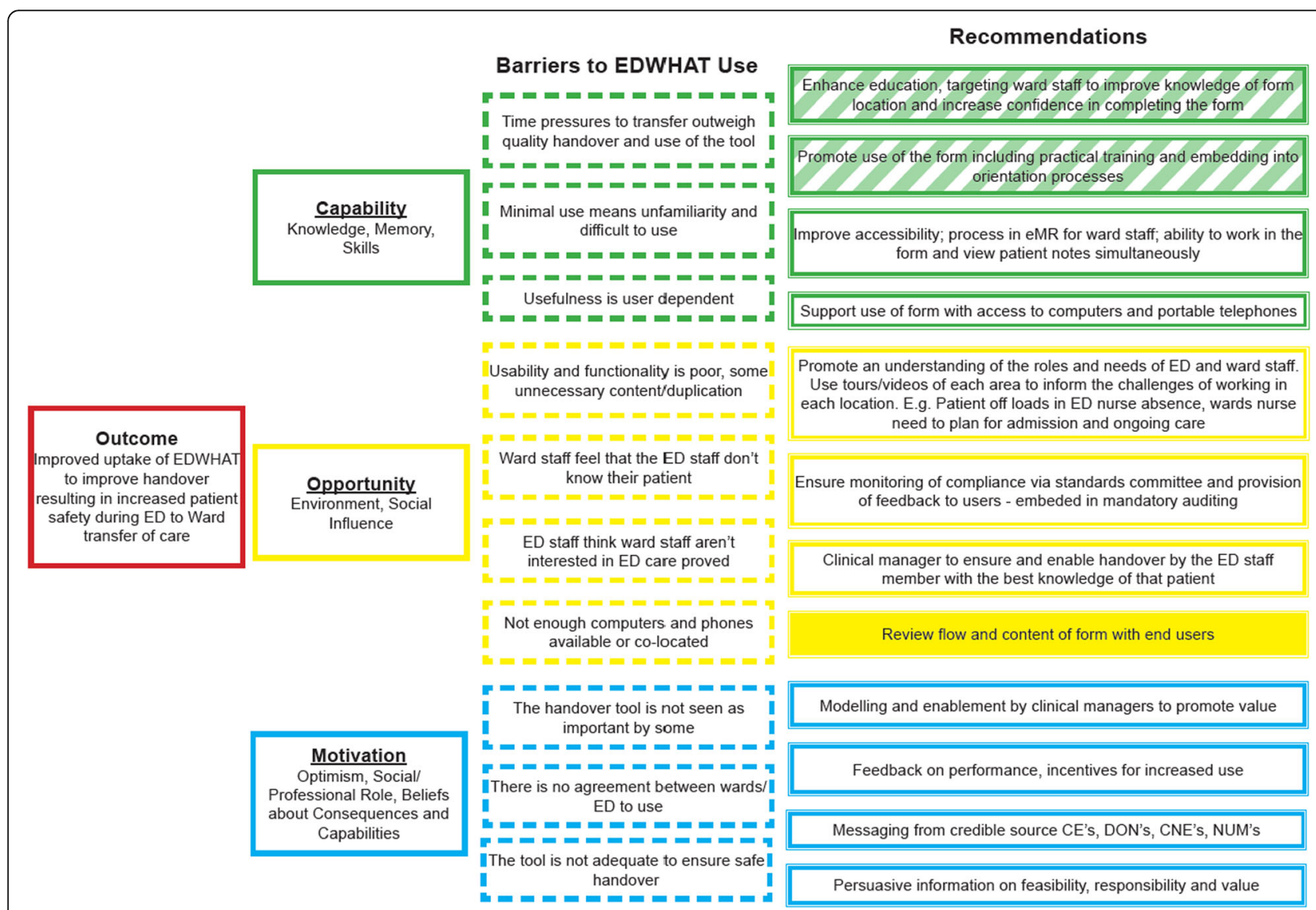

Fig. 5 Logic map demonstrating the mapping process and selection of BCTs to inform the strategy to improve uptake of the ED to Ward Handover Tool. *Filled boxes or partially filled boxes in the recommendations column represent BCTs that were completed or partially completed in the original implementation 


\section{Prior to implementation}

$\square$ Development of a working group with representation from management, handover practice standards committee members, wards and emergency department. Consider the inclusion of specialist areas such as paediatrics mental health, maternity and critical care areas

Review the content of the tool to ensure compliance with local policy, variants can be created within the tool, such as paediatric specific information.

Identify any potential infrastructure gaps such as availability of phones and computers

Consult with local eMR support team to generate within eMR and ensure functionality that includes the ability to view multiple screens during the handover process

Develop an education role out plan to capture staff from all areas. Education should include:

- why the form is important, such as critical incident numbers related to hand over

- the people who will support the implementation, such as managers and clinical specialists

- what information can be expected to be handed over

- how to escalate concerns if problems occur during implementation

- the needs and expectations of the giver and receiver of handover

- considerations from the logic map / recommendations of the EDWHAT study

- Train the trainer to ensure fidelity of messaging

Set "go live" date

Provide training prior to implementation and develop support process for both ED and ward staff for the initial period of go live

Review at regular intervals during roll out, feedback to staff

Review implementation, make any changes to tool and process if required

Embed into normal practice through orientation processes

Ongoing monitoring and feedback to ensure sustainability

$\square$ Incidents related to handover continue to be reviewed and reported to standards committee

Standard committee to monitor use of form and provide feedback to teams as required

Tool updates available at regular intervals to accommodate changes to practice and

improvements in technology

Bimonthly compliance audit and feedback to relevant stakeholders

Fig. 6 Implementation checklist

perhaps the Promoting Action on Research Implementation in Health Services Framework or PARIHS Framework [32]; however, human behaviour, which is central to the sustainable success of any change, must also be incorporated. Using a systematic four-step approach as the principal framework to inform intervention development process such as that proposed by French et al. [15] is ideal and is known to be effective in the acute care context [19]. Colquhoun et al.'s [33] systematic review of papers focussing on methods for designing interventions to change health care professionals' behaviour reported that designing an intervention for individual-level change includes identifying barriers, selecting intervention components, using theory and engaging end-users. Developing interventions systematically based on evidence and theory enhances implementation success and sustainable worthwhile effect. Further, clear presentation of the methods of theory use progresses theory development by helping to understand why interventions have failed or succeeded [34].

All intervention functions, with the exception of environmental restructuring and financial incentives, were 
assessed as affordable and practical within existing roles and orientation processes. Environmental restructuring was ranked as one of the most important interventions required, and while some investment will be required, the majority can be achieved with existing IT and senior nursing staff roles. Additional computers are not an onerous budget item and a priority. When operationalising the reinforcement and persuasive interventions, particular care must be taken to ensure an equitable approach to all staff to ensure no staff feel "singled out" and the intervention is not misconstrued as too authoritarian. A transformational leadership approach is preferred [35]. The success of the interventions would also be enhanced by nursing staff from different areas having a shared mental model, that is, a common understanding of the task (handover process) and the clinical and logistical needs to conduct handover for their colleague [36]. It would also ensure that nursing staff from different areas of the hospital are familiar with one another's roles and responsibilities, could anticipate each other's needs and be flexible [37].

In relation to education as a method to influence change, the excessive educational focus may lead to neglect in other domains and in isolation is unlikely to be effective in increasing uptake of the EDWHAT [38]. This is reinforced by the fact that education was the main intervention in initial implementation that had suboptimal uptake. The study process also highlights the importance of using a structured, evidence-informed process to diagnose implementation problems and solutions that are likely acceptable and practical to the organisation to enable success. This is demonstrated in the logic map (Fig. 5). The initial implementation of the EDWHAT partially considered only three aspects of human behaviour (knowledge, skills and beliefs about capability). The poor uptake of the tool is a clear indication that for interventions to success in the clinical environment, influences on human behaviour must be addressed. A re-implementation strategy is now under consideration by the organisation; however, it must be adequately resourced and take into consideration the findings of this study for optimal implementation. It will be necessary to evaluate the re-implementation of the tool in the future.

\section{Limitations}

There were some limitations to this study. Firstly, the survey response rate was much lower than expected (10\%) despite allowing time during work hours to complete the survey, although data saturation was reached. The overall estimate of staff obtained from departmental managers included part-time, casual and visiting staff, as well as those on maternity, annual and long service leave which may have exaggerated the actual numbers of staff available to participate. Staff from all departments and levels of seniority completed the survey, resulting in a representative sample.

The theoretical frameworks provided a systematic method for the identification of facilitators and barriers; however, our experience was of crossover between domains. These were resolved with a discussion between the authors. The authors agreed to categorise barriers or facilitators in one domain over another to aide in the integration and interpretation of the results while appreciating the potential interaction between TDF domains.

\section{Conclusions}

To improve uptake, the functionality, content, and flow of the handover tool must be revised, alongside environmental restructuring. Modelling and enabling of effective handover by nursing management is also required. Nurses need increased awareness of each other's needs to develop a shared mental model, and monitoring and enforcement of tool use should become part of a routine audit.

\section{Supplementary information}

Supplementary information accompanies this paper at https://doi.org/10. 1186/s43058-020-00045-1.

\section{Additional file 1. Supplementary Material 1. STROBE \\ Statement-Checklist of items that should be included in reports of cross-sectional studies}

Additional file 2. Supplementary Material 2. Questionnaire used for EDWHAT.

\section{Abbreviations}

APEASE: Affordable, Practical, Effective, Acceptable, had Side-effects and were safe and Equitable; BCTs: Behaviour change techniques; BCTTv1: Behaviour Change Technique Taxonomy; btf: Between the flags; ED: Emergency department; EDWHAT: ED to Ward Handover Tool; eMR: Electronic medical record; ISLHD : Illawarra Shoalhaven Local Health District; obs: Observations; PARIHS Framework: Promoting Action on Research Implementation in Health Services Framework; REDCap: Research Data Capture; TDF: Theoretical Domains Framework; UOW: University of Wollongong

\section{Acknowledgements}

Nil

Authors' contributions

$\mathrm{KR}$ and $\mathrm{KC}$ conceived and designed the study. $\mathrm{KC}$ secured funding. $\mathrm{KC}, \mathrm{KR}$, and TE developed the study protocol. TE built the study instruments. KR supervised data collection. TE and ME analysed the quantitative data. KC and KR performed qualitative analysis and integration. All authors prepared the manuscript. The authors read and approved the final manuscript.

\section{Funding}

This study was supported by the System Purchasing Branch, NSW Ministry of Health.

\section{Availability of data and materials}

Questionnaire used, qualitative analysis and mapping are available as supplementary files (please see additional files).

\section{Ethics approval and consent to participate}

Research conducted as part of this study adhered to the National Statement on the Conduct of Human Research by the Australian National Health and Medical Research Council and was approved by the University of 
Wollongong (UOW) and ISLHD Health and Medical Human Research Ethics Committee (LNR/18/WGONG/148). Participants were invited to participate via email forwarded on from their relevant department manager. The email sent included the participant information and consent form clearly stating the voluntary nature of the study, risks and confidentiality. Participation in the survey was voluntary and anonymous.

\section{Consent for publication}

Not applicable

\section{Competing interests}

The authors declare that they have no competing interests.

\section{Author details}

${ }^{1}$ Illawarra Shoalhaven Local Health District, Wollongong Hospital, Loftus Street, Wollongong NSW 2500, Australia. ${ }^{2}$ Susan Wakil School of Nursing and Midwifery, Sydney Nursing School, Faculty of Health and Medicine, The University of Sydney, 88 Mallett Street, Camperdown, NSW 2006, Australia ${ }^{3}$ Illawarra Health and Medical Research Institute, Building 32, University of Wollongong, Northfields Ave, Keiraville, NSW 2522, Australia.

\section{Received: 28 October 2019 Accepted: 5 June 2020}

\section{Published online: 27 August 2020}

\section{References}

1. NSW Health. Implementation toolkit: standard key principles for clinical handover. In: Acute Care Taskforce, ed. Vol ISBN 978174187412 9. North Sydney 2009:1-43.

2. Australian Commission on Safety and Quality in Health Care. The NSQHS standards, communicating for safety standard, communication at clinical handover action 6.4b. In. Canberra 2019

3. WHO Collaborating Centre for Patient Safety Solutions. Communication During patient Hand-Overs. 2007. https://www.who.int/patientsafety/topics/ solutions/en/. Accessed 20 June 2020

4. Manias E, Gerdtz M, Williams A, Dooley M. Complexities of medicines safety: communicating about managing medicines at transition points of care across emergency departments and medical wards. J Clin Nurs. 2015:24(1-2):69-80.

5. Arora V, Johnson J, Lovinger D, Humphrey HJ, Meltzer DO. Communication failures in patient sign-out and suggestions for improvement: a critical incident analysis. Quality \& safety in health care. 2005;14(6):401-7.

6. Eggins S, Slade D. Communication in clinical handover: improving the safety and quality of the patient experience. J Public Health Res. 2015;4(3):666.

7. Australian Institute of Health and Welfare. Emergency department care 2017-18: Australian hospital statistics. Canberra: AlHW; 2018.

8. Kilcoyne M, Dowling M. Working in an overcrowded accident and emergency department: nurses' narratives. Aust J Adv Nurs. 2007;25(2):21-7.

9. Andrew MS, Jeffrey AC, Jeffrey SY, Britt LD, et al. Young versus old: factors affecting mortality after blunt traumatic injury/discussion. Am Surg. 2002; 68(11):942.

10. Anderson J, Malone L, Shanahan K, Manning J. Nursing bedside clinical handover - an integrated review of issues and tools. J Clin Nurs. 2015;24(56):662-71.

11. Ekwall A. Acuity and anxiety from the patient's perspective in the emergency department. J Emerg Nurs. 2013;39(6):534-8.

12. Wilson R. Improving clinical handover in emergency departments. Emergency nurse. 2011;19(1):22-6.

13. Porteous JM, Stewart-Wynne EG, Connolly M, Crommelin PF. iSoBAR - a concept and handover checklist: the National Clinical Handover Initiative. Med J Aust. 2009:11(1):S152-6.

14. Creswell J, Piano CV. Designing and conducting mixed methods research. Thousand Oaks, CA: Sage Publications; 2011

15. French S, Green S, O'Connor D, et al. Developing theory-informed behaviour change interventions to implement evidence into practice: a systematic approach using the Theoretical Domains Framework. Implementation Science. 2012;7(38).

16. Curtis K, Munroe B, Van C, Elphick T. The implementation and usability of HIRAID, a structured approach to emergency nursing assessment. Australasian Emergency Care. 2019;in press.

17. Kourouche S, Buckley T, Van C, Munroe B, Curtis K. Designing strategies to implement a blunt chest injury care bundle using the behaviour change wheel: a multi-site mixed methods study. BMC Health Serv Res. 2019;19(1):461.
18. Atkins $L$, Francis J, Islam $R$, et al. A guide to using the theoretical domains framework of behaviour change to investigate implementation problems. Implement Sci. 2017;12(1):77.

19. Curtis K, Van C, Lam M, et al. Implementation evaluation and refinement of an intervention to improve blunt chest injury management-a mixedmethods study. J Clin Nurs. 2017.

20. Michie S, Atkins L, West R. The behaviour change wheel: a guide to designing interventions. Great Britain: Silverback Publishing; 2014.

21. Braun V, Clarke V. Using thematic analysis in psychology. Qual Res Psychol. 2006;3.

22. Graneheim UH, Lundman B. Qualitative content analysis in nursing research: concepts, procedures and measures to achieve trustworthiness. Nurse Educ Today. 2004;24(2):105-12.

23. Amemori M, Michie $\mathrm{S}$, Korhonen T, Murtomaa H, Kinnunen TH. Assessing implementation difficulties in tobacco use prevention and cessation counselling among dental providers. Implement Sci. 2011;6.

24. Murphy M, Curtis K, McCloughen A. Facilitators and barriers to the clinical application of teamwork skills taught in multidisciplinary simulated trauma team training. Injury. 2019;50(5):1147-52.

25. Michie $S$, Atkins L, West R. The behaviour change wheel-a guide to designing interventions. Great Britain: Silverback Publishing; 2014.

26. Sinnott C, Mercer SW, Payne RA, Duerden M, Bradley CP, Byrne M. Improving medication management in multimorbidity: development of the MultimorbiditY COllaborative medication review and DEcision making (MY COMRADE) intervention using the behaviour change wheel. Implement Sci. 2015;10(1):132.

27. Michie S, van Stralen M, West R. The behaviour change wheel: a new method for characterising and designing behaviour change interventions. Implement Sci. 2011;6:42.

28. Michie S, Richardson M, Johnston $M$, et al. The behavior change technique taxonomy ( $\mathrm{v} 1$ ) of 93 hierarchically clustered techniques: building an international consensus for the reporting of behavior change interventions. Ann Behav Med. 2013;46(1):81-95.

29. Titler M. The evidence for evidence-based practice implementation. In: Hughes $\mathrm{R}$, editor. Patient safety and quality: an evidence-based handbook for nurses. Rockville (MD): Agency for Healthcare Research and Quality; 2008.

30. Bate P, Mendel P, Robert G. Organizing for quality: the improvement journeys of leading hospitals in Europe and the United States: Radcliffe Publishing; 2008.

31. Schaffer MA, Sandau KE, Diedrick L. Evidence-based practice models for organizational change: overview and practical applications. J Adv Nurs. 2013;69(5):1197-209.

32. Rycroft-Malone J. The PARIHS framework-a framework for guiding the implementation of evidence-based practice. J Nurs Care Qual. 2004;19(4):297-304.

33. Colquhoun HL, Squires JE, Kolehmainen N, Fraser C, Grimshaw JM. Methods for designing interventions to change healthcare professionals' behaviour: a systematic review. Implement Sci. 2017;12(1):30.

34. O'Cathain A, Croot L, Sworn K, et al. Taxonomy of approaches to developing interventions to improve health: a systematic methods overview. Pilot Feasibility Stud. 2019;5:41.

35. Cheng C. Transformational leadership and social identity as predictors of team climate, perceived quality of care, burnout and turnover intention among nurses. Pers Rev. 2016;45(6):1200-16.

36. Jonker CM, van Riemsdijk MB, Vermeulen B. Shared mental models. 2011; Berlin. Heidelberg. .

37. Courtenay M, Nancarrow S, Dawson D. Interprofessional teamwork in the trauma setting: a scoping review. Hum Resour Health. 2013;11(1):57.

38. Forsetlund L, Bjorndal A, Rashidian A, et al. Continuing education meetings and workshops: effects on professional practice and health care outcomes. Cochrane Database Syst Rev. 2009;2:CD003030.

\section{Publisher's Note}

Springer Nature remains neutral with regard to jurisdictional claims in published maps and institutional affiliations. 